\title{
Biofilm detection in natural unconsolidated porous media using a low-field magnetic resonance system
}

\author{
Authors: Alexis B. Sanderlin, Sarah J. Vogt, Elliot \\ Grunewald, Bridget A. Bergin, and Sarah L. Codd
}

NOTICE: This document is the unedited author's version of a Submitted Work that was subsequently accepted for publication in Environmental Science \& Technology, copyright (C) American Chemical Society after peer review. To access the final edited and published work, see http://dx.doi.org/10.1021/es3040686.

Sanderlin AB, Vogt SJ, Grunewald E, Bergin BA, Codd SL, "Biofilm detection in natural unconsolidated porous media using a low-field magnetic resonance system," Environmental Science \& Technology, April 2013 47(2): 987-992. 


\title{
Biofilm Detection in Natural Unconsolidated Porous Media Using a Low-Field Magnetic Resonance System
}

\author{
Alexis B. Sanderlin, ${ }^{\dagger, \ddagger}$ Sarah J. Vogt, ${ }^{\dagger \dagger}$ Elliot Grunewald, ${ }^{\|}$Bridget A. Bergin, ${ }^{\ddagger}$, and Sarah L. Codd $^{\ddagger, \S, *}$ \\ ${ }^{\dagger}$ Department of Chemical and Biological Engineering, Montana State University, Bozeman, MT \\ ${ }^{\ddagger}$ Center for Biofilm Engineering, Montana State University, Bozeman, MT \\ ${ }^{\S}$ Department of Mechanical and Industrial Engineering, Montana State University, Bozeman, MT \\ "Vista Clara Inc., 12201 Cyrus Way Ste. 104, Mukilteo, WA
}

\begin{abstract}
The extent to which $T_{2}$ relaxation measurements can be used to determine biofouling in several natural geological sand media using a low-field $(275 \mathrm{kHz}, 6.5 \mathrm{mT})$ NMR system has been demonstrated. It has been previously shown that, at high laboratory strength fields $(300 \mathrm{MHz}, 7 \mathrm{~T}), \mathrm{T}_{2}$ techniques can be used as a bioassay to confirm the growth of biofilm inside opaque porous media with low magnetic susceptibilities such as borosilicate or soda lime glass beads. Additionally decreases in $T_{2}$ can be associated with intact biofilm as opposed to degraded biofilm material. However, in natural geological media, the strong susceptibility gradients generated at high fields dominated the $T_{2}$ relaxation time distributions and biofilm growth could not be reliably detected. Samples studied included Bacillus mojavensis biofilm in several sand types, as well as alginate
\end{abstract}

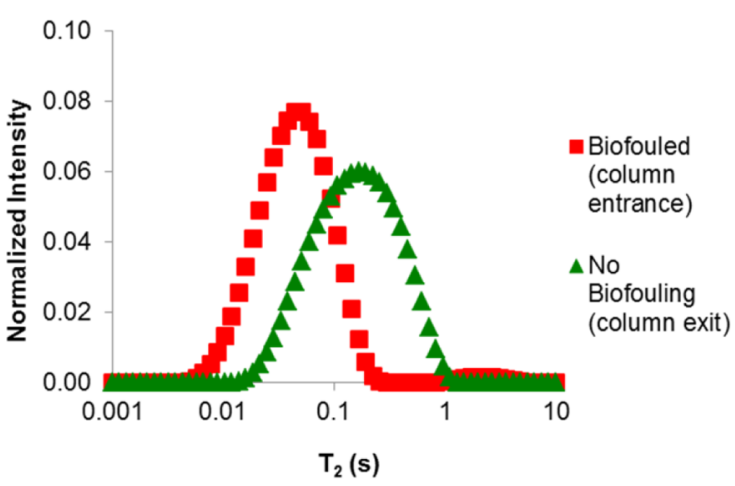
solution and alginate gel in several sand types. One of the sand types was highly magnetic. Data was collected with a low-field $(275 \mathrm{kHz}, 6.5 \mathrm{mT})$ benchtop NMR system using a CPMG sequence with an echo time of $1.25 \mathrm{~ms}$ providing the ability to detect signals with $T_{2}$ greater than $1 \mathrm{~ms}$. Data presented here clearly demonstrate that biofilm can be reliably detected and monitored in highly magnetically susceptible geological samples using a low-field NMR spectrometer indicating that low-field NMR could be viable as a biofilm sensor at bioremedation sites.

\section{INTRODUCTION}

Bioremediation, the use of bacteria to remove or contain pollutants and waste in the subsurface, is being used as a means to control contaminants in the environment. ${ }^{1-5}$ However, the monitoring of bacterial growth comes with several challenges. Direct sampling, the method most often used to determine the efficacy of the bioremediation process, provides little spatial information, can be quite costly and potentially alters the environment it is intended to measure. Additionally, the samples collected from contaminated sites pose significant safety, regulatory, and cost issues. Nuclear magnetic resonance, NMR, has been proposed as an effective means to nondestructively monitor biofouling on-site in the subsurface. ${ }^{6,7}$

Hydrodynamics and mass transport inside biofouled porous media has long been of interest due to its broad importance not only in bioremediation but also in nature, industry, and medicine. ${ }^{8}$ Since the early 1990s, researchers have used a variety of porous systems in the lab (e.g., sand, glass bead packs, rocks, and flat plate channel reactors) to measure how the extracellular polymeric substance (EPS) production from bacterial cells changes a system's hydraulic conductivity, mass transport, and dynamic flow patterns. ${ }^{2,6,9}$ Some of these lab techniques have also been applied in large bioremediation field scale studies to determine biofilm impact and presence. ${ }^{10}$ Using high magnetic field NMR to study how biofouling affects transport in porous media is a relatively new application., 6,11-14 High-field NMR imaging has been used to spatially resolve biofouled porous systems, monitor flow changes in biofouled bead packs, and detect the extent of biofouling in bead packs. ${ }^{6,12,15,16}$ However, no previous studies have successfully applied NMR to study biofilms in natural porous media, even in the lab.

This article presents the first use of low-field NMR to study biofilms in porous media and indicates it may be a viable technique for monitoring biofilm growth at bioremediation sites. It has been previously shown that laboratory high-field NMR measurements can clearly be used as a bioassy to confirm the presence of biofouling in model porous media and obtain complementary and unique information about biofilms, but so far the challenge of dealing with high magnetic susceptibility gradients has limited the application in natural porous media. Specifically, the extent of biofouling in natural geological materials cannot be determined using high-field NMR due to the strong susceptibility gradients produced by the magnetic materials present in geological samples. ${ }^{7}$ These susceptibilities 
dominate the $T_{2}$ distributions hindering the detection of biofouling. These magnetic field gradients in natural sediments and rocks are due to the presence of magnetically susceptible minerals (such as iron-bearing minerals) within the grains or on the grain surfaces. The presence of magnetic minerals causes local variations of magnetic flux in an otherwise uniform static magnetic field and generates spatial gradients in the static field on a very small scale. These gradients cause an irreversible loss of coherence of the NMR signal and hence a more rapid decay of the detected signal due to diffusion (random movement) of water molecules in the gradient fields. ${ }^{17}$ In natural rocks and sediments, these effects associated with magnetic gradients dominate the relaxation response overwhelming the biofilm signature. To enable the sensitivity of the NMR measurement to the biofouling processes of interest, it is therefore critical to reduce the effect of magnetic susceptibility. The magnitude of magnetic susceptibility gradients generally scales linearly with the intensity of the applied static field. ${ }^{18}$ Although lowering the static magnetic field is the easiest way to reduce susceptibility gradients, the measured NMR signal also decreases with static magnetic field strength. Nonetheless, the measurements presented here indicate low-field NMR techniques can reliably detect biofouling in porous materials even with strong magnetic components establishing the method as a potential in situ subsurface biosensor.

A low-field $(6.5 \mathrm{mT}, 275 \mathrm{kHz})$ benchtop NMR system with an open bore was used in this study to ascertain the extent to which $T_{2}$ relaxation measurements can be used to measure biofouling in several natural geological sand media. This lowfield spectrometer is approximately 3 orders of magnitude lower than the 2-7 $\mathrm{T}(85-300 \mathrm{MHz})$ NMR spectrometers that have typically been used to characterize NMR properties of biofilms in porous media. ${ }^{6,7,12-14}$ In this study, a Bacillus mojavensis biofilm was grown in a large silica sand column. A range of samples intended to determine the ability of low-field NMR to sense biofilms were then constructed using biofilm, alginate solution, and alginate gels in model and natural sands (natural unconsolidated porous media). Data presented here clearly demonstrate that biofilm can be reliably detected and monitored in high magnetic content geological samples using a low-field NMR spectrometer. Such samples cannot even be safely placed in a high magnetic field due to the forces on the material. This result is significant in that it paves the way for NMR biofilm sensors using low-field systems to be extended to the field scale.

\section{THEORY}

Biofilm Formation. Biofilms form when bacterial cells adhere to a surface and begin to excrete biopolymers such as proteins, DNA, and carbohydrates that form a protective matrix collectively referred to as extracellular polymeric substance (EPS), an often slimy appearing layer encasing the bacterial cells. ${ }^{19,20}$ Variation in the nature of the EPS layer leads to biofilm thicknesses that vary from a few to several hundred micrometers depending on the microbial composition. ${ }^{21}$ Biofilms are predominant in a range of settings such as the surfaces of teeth, the walls of water treatment equipment, industrial pipes, ship hulls, and human implant surfaces. ${ }^{22}$ They are also found in the subsurface where complex ecosystems of microbial species coexist and can help or hinder processes such as oil recovery and bioremediation. ${ }^{3,23}$ Biological activity can interact with the geochemistry precipitation of contaminants, whereas the presence of EPS can impact the flow dynamics of subsurface liquids. ${ }^{24}$ The recovery of oil from or the remediation of contaminants within porous earth formations can be strongly impacted by bioactivity, as the subsurface porous media is in essence a large packed bed bioreactor. ${ }^{3,5,25}$

$T_{2}$ Relaxation. NMR active spins in a sample placed into a magnetic field $B_{\mathrm{o}}$ will align both parallel and antiparallel to the magnetic field with a small number of spins more likely aligned parallel to the field. This spin distribution is governed by Boltzmann statistics and the slightly larger magnetization along the field is defined as the bulk magnetization, $M_{0}$. An NMR experiment starts with disturbing the magnetization of the sample from equilibrium using a radio frequency (RF) pulse applied at the Larmor frequency, $\omega_{0}=\gamma B_{0}$, where $\gamma$ is the gyromagnetic ratio. The NMR signal will relax back to equilibrium on two different time scales, known as $T_{1}$ and $T_{2}$. The $T_{2}$ relaxation, which is of importance in these experiments, is due to dipolar spin-spin interactions.

Factors such as inhomogeneous magnetic fields, diffusion, and surface interactions that act on a time scale longer than hundreds of microseconds and cause inhomogeneous dephasing can be refocused by applying a 180 degree pulse at time $\tau$ after the initial 90 degree excitation to form what is termed a spin echo. Molecular interactions that occur on time scales shorter than the echo time, such as surface interactions, will not be refocused and will reduce the measured value of $T_{2}$ for those surface impacted molecules. Repetition of $n 180$ degree pulses spaced every $2 \tau$ results in the amplitude of the echoes $M(2 n \tau)$ decaying as:

$$
M(2 n \tau)=\sum_{T_{2}} M_{0}\left(T_{2}\right) \exp \left(\frac{-2 n \tau}{T_{2}}\right)
$$

where $M_{0}\left(T_{2}\right)$ is the distribution of the $T_{2}$ relaxation times due to the physical and chemical environment of the spins in the sample with each $T_{2}$ relaxation value. This series of 180 degree pulses is known as the Carr, Purcell, Meiboom, and Gill (CPMG) sequence. $^{26}$ By then applying an inverse Laplace transform to the detected signal, the $T_{2}$ relaxation time distribution of spins $M_{0}\left(T_{2}\right)$ within the sample is obtained. ${ }^{27}$

Spin-spin $T_{2}$ magnetic relaxation provides information on the rotational mobility of the protons on water molecules. In regions where exchangeable protons are present, such as those on the $\mathrm{OH}$ groups of biopolymers, fast proton-proton exchange between the protons on water molecules, and the protons on the biopolymers can reduce the measured $T_{2}$ relative to a measurement made in pure water. Specifically, in regions where biofouling is present, proton-proton exchange between the protons on water molecules and the protons on the cross-linked biopolymers in the EPS results in a reduced $T_{2}$ time relative to a measurement made when the water is only in the presence of the freely diffusing molecules in the growth media. $^{28}$

Low-Field Magnetic Resonance. Advances in laboratory NMR spectrometers and the associated measurement techniques have typically exploited the fact that NMR signal increases as the magnetic field is made stronger and more homogeneous. Hence, many chemistry and physics laboratories house spectrometers which operate at $300-700 \mathrm{MHz}$ due to the stable superconducting magnets that produce fields of 7-17 T. However, these strong magnetic fields are not suitable for samples that are extremely heterogeneous and/or made of materials with significant magnetic susceptibility, including common geologic media containing magnetic minerals. For this 
reason, oil companies' down-the-borehole spectrometers that use NMR in the subsurface operate at $2 \mathrm{MHz}(50 \mathrm{mT})$. These down-the-borehole spectrometers use inside out electronics relative to laboratory NMR. The electronics are on the inside of a cylindrical 5 in. diameter tool, and the NMR measurement is obtained from the region of the subsurface surrounding the tool.

Vista Clara, Inc. (Mukilteo, WA, USA), has developed a down-the-borehole tool that is designed to operate in the smaller 2 in. diameter, cased wells that are commonly used for groundwater monitoring. ${ }^{29}$ The tool operates at a lower frequency of $\sim 275 \mathrm{kHz}(6.5 \mathrm{mT})$ to detect signal from a larger surrounding region of the subsurface. The results presented here provide benchmarking data to demonstrate that a down-the-borehole tool operating at these low frequencies can potentially detect and monitor changes in the extent of biofouling in the subsurface with NMR relaxation measurements.

\section{MATERIALS AND METHODS}

Reactor Design. The reactor was constructed of schedule 40 clear polyvinyl chloride (PVC) piping having an inside diameter of 5.08 and $38.1 \mathrm{~cm}$ length. Two column reactors are shown in figure 1 . To prepare the reactor for the experiments, the column was wet-packed with silica sand (Unimin Corp 4095) and disinfected.

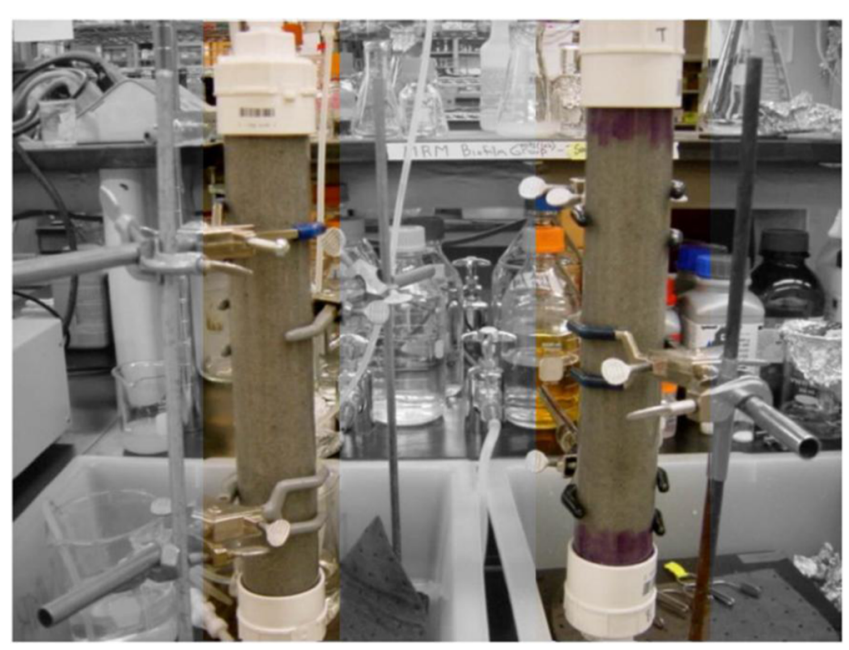

Figure 1. Silica sand column reactors used to grow biofilm.

Bacillus mojavensis, which forms a thick biofilm, was chosen due to its importance in bioremediation. ${ }^{23}$ Brain Heart Infusion [Becton, Dickinson and Company], or BHI, the broth used as the bacterial growth medium, was prepared according to the recipe of Mitchell et al. ${ }^{23}$ After inoculation with B. mojavensis, $\mathrm{BHI}$ was pumped upward through the reactor in a flow-through system at $500 \mathrm{~mL} \mathrm{hr}^{-1}$ in approximately 2 to $8 \mathrm{~h}$ pulses for 10 days. The volumetric flow rate of $500 \mathrm{~mL} \mathrm{hr}^{-1}$ corresponded to a maximum velocity of approximately $0.17 \mathrm{~mm} \mathrm{~s}^{-1}$ through the reactor, assuming a porosity of 0.4 for the sand. Throughout the growth process, a pressure gauge located at the entrance of the reactor was monitored. After observing a large pressure increase at the reactor inlet, the flow system was disassembled and the samples prepared for NMR analysis. Sand samples were collected from both the bottom (column entrance) and top (column exit) of the reactor. It was evident that biofouling primarily occurred at the entrance to the reactor, as seen in Figure 2. The sample from the entrance of the reactor where
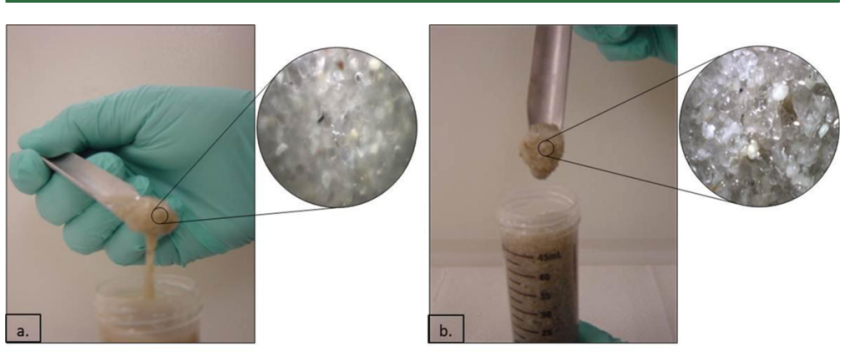

Figure 2. Sand samples from (a) the entrance (biofouled) of the reactor and (b) from the exit (not biofouled) of the reactor. The biofouling clearly occurred at the entrance of the reactor.

the BHI was being pumped into the reactor was completely biofouled, exhibiting a gelatinous consistency and cloudy color (part a of Figure 2). In contrast, the sample from the exit of the reactor showed no signs of biofouling and the sand displayed characteristics similar to wet beach sand (part b of Figure 2).

In addition to the biofilm growth in the silica sand column, biofilm mixed with samples of sand taken the Hanford Buried Waste Test Facility in Richland, Washington, were also analyzed. The Hanford site sand was less homogeneous in size than the silica sand and contained significant iron impurities. The silica sand and Hanford sand represented the two extremes of geological media with regard to magnetic content.

Sand Analysis. The silica sand used in the column reactor had the following grain size distribution: $28.5 \%>840 \mathrm{um}$ diameter, $38.1 \%$ between 595 um and 840 um, 32.2\% between 354 um and 595 um, $1.2 \%<354$ um diameter. A neodynium magnet was not able to move any of the silica sand. Mixing the silica sand with $\mathrm{HCl}$ did not produce any bubbles indicating there were no calcium ions present.

The Hanford sand had the following grain size distribution: $11.8 \%>840$ um diameter, $13.4 \%$ between 595 um and 840 um, $42.3 \%$ between $354 \mathrm{um}$ and $595 \mathrm{um}, 17.2 \%$ between $177 \mathrm{um}$ and 354 um, 9.0\% between 100 um and 177 um diameter, 5.3\% between 50 um and 100 um diameter, $1 \%<50$ um diameter. A neodymium magnet was able to pick up and move $65 \%$ of the sand demonstrating how strongly magnetic this sand was. Mixing the Hanford sand with a $0.1 \mathrm{M} \mathrm{HCl}$ solution did not produce any bubbles; however, mixing with a $1.0 \mathrm{M} \mathrm{HCl}$ solution did, indicating the presence of calcium ions.

Alginate Gel Preparation. Biofilms are primarily composed of a matrix of bacterially produced polymers and proteins known as the extracellular polymeric substance (EPS). ${ }^{19}$ Alginate is a biopolymer isolated from both brown algae and from certain types of bacterial biofilms. ${ }^{30,31}$ Alginate solutions and gels are often used as model systems for biofilms, ${ }^{32}$ so they were also analyzed to study the contribution to the $T_{2}$ relaxation caused by the mobility of the alginate biopolymer molecules in the solution or gel phase. ${ }^{33}$

Algal alginate [Acros Organic] solutions were prepared at 2 wt $\%, 1 \mathrm{wt} \%$, and $0.5 \mathrm{wt} \%$ alginate in distilled water in $5.7 \mathrm{~cm}$ diameter, clear plastic bottles. The solutions were then gelled using a diffusion reaction mechanism by adding a solution of $0.5 \mathrm{M} \mathrm{CaCl}_{2}$ on top of the alginate solution. After gelation, the water around the gel was drained and replaced by fresh alginate solution. This procedure was followed to analyze the difference between solution and gel at the same solution concentration of 
polymer. Mixtures of $1 \mathrm{wt} \%$ alginate solution and the two different types of sand, silica, and Hanford were also prepared and then gelled using the same procedure.

Prior to mixing the Hanford sample with alginate, the sample bottle was wet-packed with an appropriate amount of Hanford sand to completely fill the bottle. The sand was removed from the bottle and mixed with the alginate solution. After combining the sand and alginate solution, only $74 \%$ of the original mass of sand could be repacked in the bottle due to partial gelation of alginate and related increased viscosity resulting in a less densely packed sample compared to the clean Hanford sand. The presence of the calcium ions and the $1 \%$ of particles less than $50 \mu \mathrm{m}$ contributed to this observed partial gelation.

NMR Measurements. NMR measurements for all samples were acquired using the portable benchtop Corona system [Vista Clara, Inc.], with an operating frequency of $\sim 275 \mathrm{kHz}$. For all experiments the ${ }^{1} \mathrm{H}$ nuclei were excited. The geometry of the magnets produced a field that was uniform over a spheroidal volume that had an average dimension of $5 \mathrm{~cm}$. Each sample was placed in the same location in the instrument for accurate and comparable measurements. Placement was checked by the reproducibility of the measurement for a standard water sample. A CPMG measurement sequence was used to assess the $T_{2}$ relaxation behavior of the samples. For each experiment, a repetition time of $10 \mathrm{~s}$, excitation pulse length of $100 \mu \mathrm{s}$, echo spacing of $1.25 \mathrm{~ms}, 800$ echoes, and 300 averages was selected.

For comparison of data from a high-field magnetic system, some of the same samples were also analyzed in a Bruker Avance $300 \mathrm{MHz}$ vertical magnet spectrometer. The Hanford sand samples contained materials that were highly magnetic and therefore could not be placed in the strong magnetic field. The silica sand and alginate solutions and gels were measured using the same NMR parameters as were used on the low-field system but with 64 averages. All of the data was processed using inverse Laplace transform ILT software developed in Matlab (MathWorks, Inc.).

\section{RESULTS AND DISCUSSION}

Silica Sand with Biofilm. In Figure 3, we show $T_{2}$ relaxation-time distributions for the low-field NMR measurements on the sand column reactor. The biofouled silica sand exhibited a $T_{2}$ time of approximately half that of the nonbiofouled sand, $450 \mathrm{~ms}$ versus $900 \mathrm{~ms}$. We attributed the

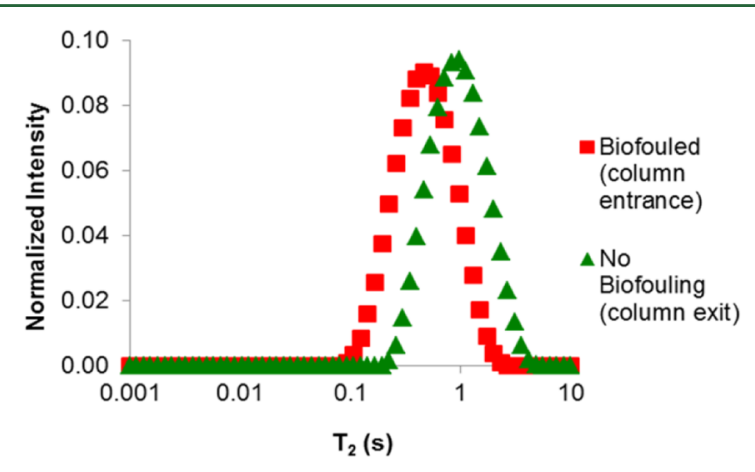

Figure 3. Silica Sand, Low Field. $T_{2}$ relaxation time distributions from the low-field NMR system showing a clear distinction between the biofouled and nonbiofouled sand. shorter relaxation time of the biofouled sand to proton-proton exchange with the biopolymers present in the biofilm EPS.

Both sand samples were also tested in the high-field (300 $\mathrm{MHz}$ ) NMR system producing analogous results as with the low-field system. Figure 4 shows that the biofouled silica sand

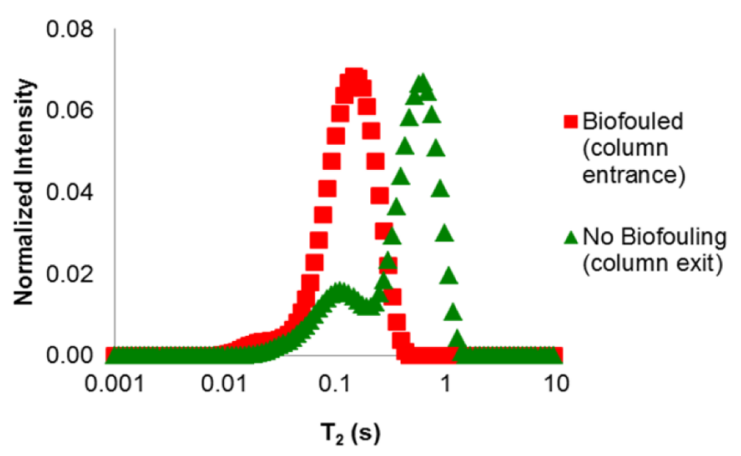

Figure 4. Silica Sand, High Field. $T_{2}$ relaxation time distributions from the high-field NMR system showing a similar distinction between the biofouled and nonbiofouled sand as seen in Figure 3. The appearance of a second shorter $T_{2}$ was due to the magnetic field gradients induced at the edges of the pores in the high field.

sample had a lower $T_{2}$ relaxation time than the nonbiofouled sample similar to the findings in Figure 3. The relaxation time in Figure 4 was lower for both samples due to the increased impacts of magnetic field susceptibility at high magnetic fields. The silica sand had low enough magnetic susceptibility that biofouling could be measured by NMR at high magnetic fields, as seen in Figure 4.

Hanford Sand with Biofilm. Biofouled silica sand was mixed with Hanford site sand in a 50:50 ratio to prepare samples that had biofilm and a high magnetic susceptibility material. Figure 5 shows that even in the presence of very high magnetic susceptibility sand, the changes in $T_{2}$ due to the biofilm polymer were significant and easily detectable at 275 $\mathrm{kHz}$.

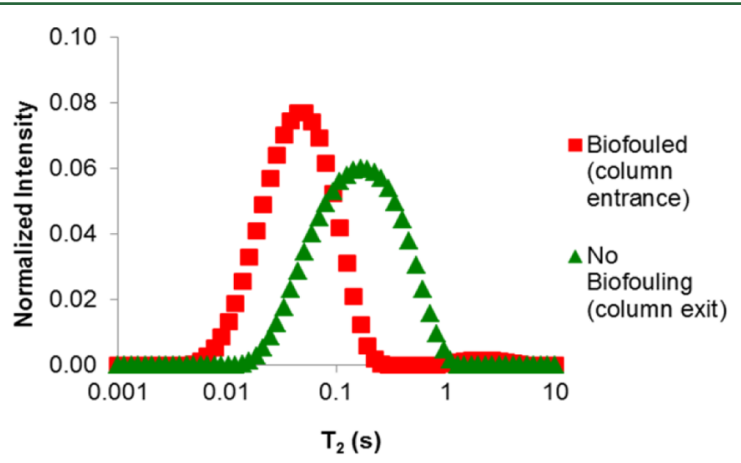

Figure 5. Silica Sand Mixed with Hanford Sand, Low Field. $T_{2}$ relaxation time distributions from the low-field NMR system showing a difference between the biofouled and nonbiofouled silica and Hanford site mixed sand samples.

Alginate Gels and Sand with Alginate Solutions. The $T_{2}$ relaxation time distributions for alginate gel and the surrounding alginate solution for two different concentrations of alginate are shown in Figure 6. As the calcium ions formed a physical gel, the proton-proton exchange between the water molecules and the more rigid polymers of the gel resulted in the lower $T_{2}$ relaxation time. The samples contained both 


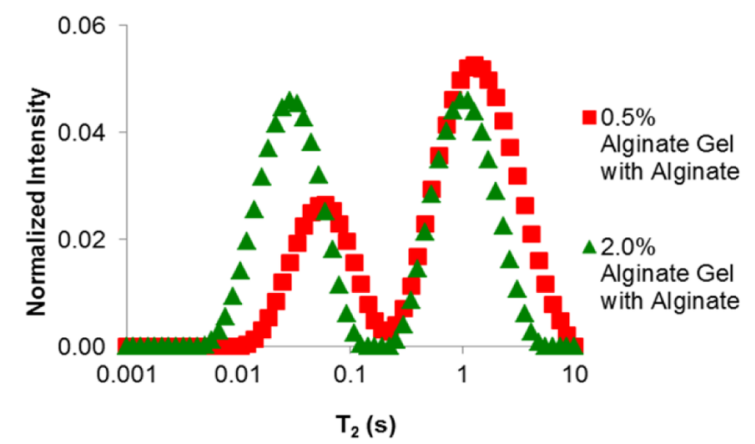

Figure 6. Alginate Gel in Silica Sand, Low Field. $T_{2}$ relaxation time distributions of the different alginate gel concentrations. Each sample contained the gelled alginate and alginate solution. For each sample, the longer $T_{2}$ resulted from the presence of the alginate solution and the shorter $T_{2}$ from the gel. The alginate solution surrounding the gel gave a peak at about $1 \mathrm{~s}$, which was the same as the solution prior to the gelling.

gelled alginate and alginate solution, which exhibited distinct $T_{2}$ times. The $T_{2}$ of the ungelled alginate solution was approximately $1 \mathrm{~s}$ in both samples (compared to a $T_{2}$ of $2 \mathrm{~s}$ for distilled water). After gelation, however, both concentrations showed the gel having a $T_{2}$ below $100 \mathrm{~ms}$, with the 2 wt $\%$ alginate gel exhibiting a shorter $T_{2}$ than $0.5 \mathrm{wt} \%$ alginate gel. A higher percentage of alginate produced a stiffer gel and provided additional proton exchange sites on the more rigid alginate polymers, resulting in a lower $T_{2}$.

Figure 7 shows the $T_{2}$ relaxation time distribution for silica sand with pure water, sand mixed with 1 wt \% alginate and sand

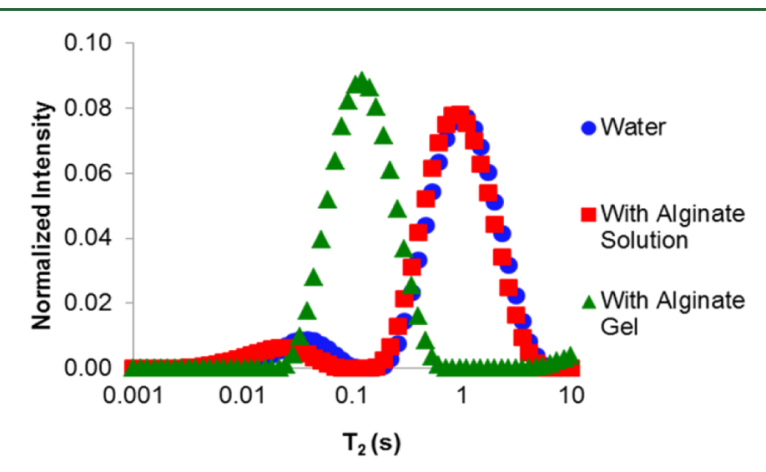

Figure 7. Alginate in Silica Sand, Low Field. $T_{2}$ relaxation time distributions of the silica sand samples using the low-field NMR system. The sand with pure water and sand with alginate solution exhibited very similar $T_{2}$ relaxation times of approximately $1 \mathrm{~s}$. However, after gelation, the $T_{2}$ got shorter, $100 \mathrm{~ms}$. This shorter $T_{2}$ was due to the proton-proton exchange of the water with the less mobile hydrogen of the biopolymer and was very similar to the interaction between the water and EPS of the biofilm seen in Figure 3.

with 1 wt $\%$ alginate after gelation. The $T_{2}$ of the sand with pure water was similar to the $T_{2}$ of the sand mixed with alginate solution. After gelation, the $T_{2}$ shifted down to approximately $100 \mathrm{~ms}$. The $T_{2}$ relaxation times in the clean silica sand and silica sand with alginate gel at low field were similar to the findings of the same sand with and without biofouling shown above in Figure 3. As the alginate gelled, the alginate biopolymer molecules became less mobile and the $T_{2}$ relaxation time shortened, similar to water interaction with the biofilm EPS. The data clearly demonstrated that it was the hydrogel phase of the biofilm which generated variation in the $T_{2}$ relaxation time distribution shown in Figure 3.

The $T_{2}$ relaxation time distributions of the 1 wt $\%$ alginate solution and gel mixed with the Hanford sand as measured in the low-field NMR system are shown in Figure 8. Both the

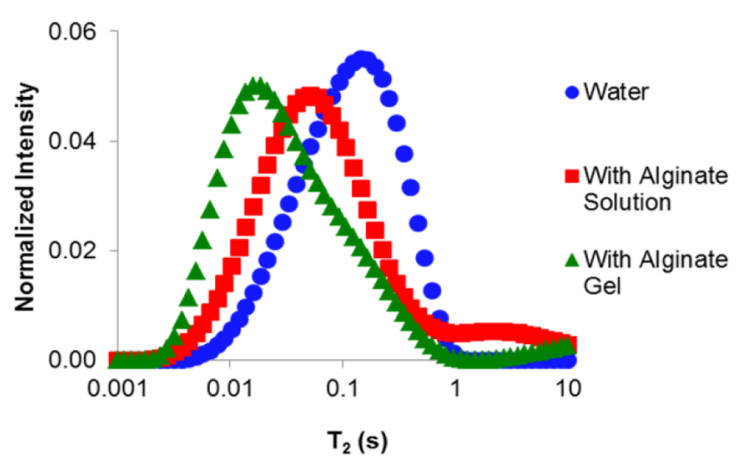

Figure 8. Alginate in Hanford Sand, Low Field. $T_{2}$ relaxation time distributions of the Hanford sand samples using the low-field NMR system. Because of the presence of iron in the Hanford sand sample, the $T_{2}$ of the sand with pure water sample was lower $\left(T_{2} \sim 100 \mathrm{~ms}\right.$, Figure 7$)$ than that of the clean silica sand $\left(T_{2} \sim 1000 \mathrm{~ms}\right)$. The Hanford sand also had charged mineral species that interacted with and changed the biopolymer conformations, which was exhibited by the sand with alginate solution having a lower $T_{2}$ than the sand with pure water sample. This was in contrast to the silica sand, which has similar $T_{2}$ times for both the sand with pure water and sand with alginate solution.

Hanford sand mixed with the alginate solution and the alginate gel exhibited lower relaxation times than the clean Hanford sand, with again the shortest $T_{2}$ exhibited after gelation. The significantly lower $T_{2}$ for the clean sample of Hanford sand $\left(T_{2}\right.$ $\sim 100 \mathrm{~ms})$ compared to the $T_{2}$ in the clean silica sand $\left(T_{2}\right.$ $\sim 1000 \mathrm{~ms}$, Figure 7) was indicative of significant iron content in the natural Hanford sample. It is interesting to observe that the $T_{2}$ for the alginate solution in the Hanford sand $\left(T_{2} \sim 30\right.$ ms) was very different from the $T_{2}$ of water in the Hanford sand $\left(T_{2} \sim 100 \mathrm{~ms}\right)$. This result contrasted with that presented in Figure 7 for silica sand in which the $T_{2}$ did not vary significantly between water and alginate solution in the silica sand. This difference was due to the presence of fine particulates in the Hanford sand that alter the polyelectrolyte alginate biopolymer conformations and interactions and caused partial gelation of the alginate solution. Additional evidence of the partial gelation of the alginate solution was provided when packing the sand into the sample bottle. The partial gelation of the alginate in the Hanford sand sample as described in the methods sectrion resulted in a lower $T_{2}$ compared to the clean Hanford sand. This indicated the ability of the NMR $T_{2}$ measurements to monitor not only biological activity and biofilm growth but also the interaction of biological activity and geochemistry.

The ability of NMR $T_{2}$ relaxation time distribution measurements to detect biofilm presence as a marker of biological activity in natural geological media has been demonstrated at low magnetic fields. Because of the use in down-the-borehole spectrometers and limitations of high-field characterization of magnetic materials, low-field NMR is relevant to the development of in situ subsurface NMR sensors. In comparing measurements of alginate solutions in a model silica sand and in a naturally occurring (and highly 
magnetic) sand from the Hanford site, the impact of the interaction between biopolymers and geochemistry indicate that the mineral content of the geological media significantly impacts the $T_{2}$ relaxation time distributions. These results demonstrate a significant potential for NMR relaxation measurements to monitor bioactivity and further characterize biogeochemical interactions in the subsurface. A single $T_{2}$ measurement alone will not be a direct indication of the presence of biofilm. However, an NMR sensor could be placed in the subsurface and used to monitor the growth and decay of biofouling over the duration of a bioremediation process.

\section{AUTHOR INFORMATION}

\section{Corresponding Author}

*Tel.: (406) 994 1944, fax: (406) 994 6292, e-mail: scodd@ coe.montana.edu.

\section{Notes}

The authors declare no competing financial interest.

\section{ACKNOWLEDGMENTS}

This material is based upon work supported, in part, by the Department of Energy under Grant DE-FG02-11ER90025 to Vista Clara. Any opinions, findings, and conclusions or recommendations expressed in this material are those of the author(s) and do not necessarily reflect the views of the Department of Energy. The authors would like to thank Prof. Paul Callaghan for the Matlab inverse Laplace transform program.

\section{REFERENCES}

(1) Beveridge, T. J.; Makin, S. A.; Kadurugamuwa, J. L.; Li, Z. S. Interactions between biofilms and the environment. FEMS Microbiol. Rev. 1997, 20 (3-4), 291-303.

(2) Bouwer, E. J.; Rijnaarts, H. H. M.; Cunningham, A. B.; Gerlach, R., Biofilms in porous media. In Biofilms II: Process Analysis and Applications, Bryers, J. D., Ed. Wiley-Liss, Inc: 2000; pp 123-158.

(3) Cunningham, A. B.; Sharp, R. R.; Hiebert, R.; James, G. Subsurface biofilm barriers for the containment and remediation of contaminated groundwater. Bioremediat J 2003, 7 (3), 151-164.

(4) Fang, Y. L.; Yabusaki, S. B.; Morrison, S. J.; Amonette, J. P.; Long, P. E. Multicomponent reactive transport modeling of uranium bioremediation field experiments. Geochim. Cosmochim. Acta 2009, 73 (20), 6029-6051.

(5) Lomesh, D.; Nuttall, H. E.; Al, C.; Garth, J.; Randy, H. In situ biofilm barriers: Case study of a nitrate groundwater plume, Albuquerque, New Mexico. Remediat J 2005, 15 (4), 101-111.

(6) Seymour, J. D.; Gage, J. P.; Codd, S. L.; Gerlach, R. Anomalous fluid transport in porous media induced by biofilm growth. Phys. Rev. Lett. 2004, 93 (19), 198103.

(7) Codd, S. L.; Vogt, S. J.; Hornemann, J. A.; Phillips, A. J.; Maneval, J. E.; Romanenko, K. R.; Hansen, L.; Cunningham, A. B.; Seymour, J. D. NMR relaxation measurements of biofouling in model and geological porous media. Org. Geochem. 2011, 42 (8), 965-971.

(8) Stoodley, P.; Debeer, D.; Lewandowski, Z. Liquid Flow in Biofilm Systems. Appl Environ Microb 1994, 60 (8), 2711-2716.

(9) Sharp, R. R.; Stoodley, P.; Adgie, M.; Gerlach, R.; Cunningham, A. Visualization and characterization of dynamic patterns of flow, growth and activity of biofilms growing in porous media. Water Sci. Technol. 2005, 52 (7), 85-90.

(10) Atekwana, E. A. Geophysical Signatures of Microbial Activity at Hydrocarbon Contaminated Sites: A Review. Surv. Geophys. 2010, 31 (2), 247-283.

(11) Lewandowski, Z.; Stoodley, P.; Altobelli, S. Experimental and conceptual studies on mass-transport in biofilms. Water Sci. Technol. 1995, 31 (1), 153-162.
(12) Hoskins, B. C.; Fevang, L.; Majors, P. D.; Sharma, M. M.; Georgiou, G. Selective imaging of biofilms in porous media by NMR relaxation. J. Magn. Reson. 1999, 139, 67-73.

(13) Beauregard, D. A.; Yong, P.; Macaskie, L. E.; Johns, M. L. Using non-invasive magnetic resonance imaging (MRI) to assess the reduction of $\mathrm{Cr}(\mathrm{VI})$ using a biofilm-palladium catalyst. Biotechnol. Bioeng. 2010, 107 (1), 11-20.

(14) Fridjonsson, E. O.; Seymour, J. D.; Schultz, L. N.; Gerlach, R.; Cunningham, A. B.; Codd, S. L. NMR measurement of hydrodynamic dispersion in porous media subject to biofilm mediated precipitation reactions. J. Contam. Hydrol. 2011, 120-21, 79-88.

(15) Paterson-Beedle, M.; Nott, K. P.; Macaskie, L. E.; Hall, L. D., Study of biofilm within a packed-bed reactor by three-dimensional magnetic resonance imaging. In Methods in Ezymology, 2001; Vol. 337, pp 285-305.

(16) von der Schulenburg, D. A. G.; Akpa, B. S.; Gladden, L. F.; Johns, M. L. Non-invasive mass transfer measurements in complex biofilm-coated structures. Biotechnol. Bioeng. 2008, 101 (3), 602-608.

(17) Keating, K.; Knight, R. A laboratory study to determine the effect of iron oxides on proton NMR measurements. Geophysics 2007, 72 (1), E27-E32.

(18) Callaghan, P. T. Principles of Nuclear Magnetic Resonance Microscopy; Oxford University Press: New York, 1991.

(19) Costerton, J. W. Introduction to biofilm. Int. J. Antimicrob. Ag. 1999, 11 (3-4), 217-221.

(20) Sutherland, I. W. Biofilm exopolysaccharides: A strong and sticky framework. Microbiology-(UK) 2001, 147, 3-9.

(21) Nivens, D. E.; Ohman, D. E.; Williams, J.; Franklin, M. J. Role of alginate and its $\mathrm{O}$ acetylation in formation of Pseudomonas aeruginosa microcolonies and biofilms. J. Bacteriol. 2001, 183 (3), 1047-1057.

(22) Characklis, W. G.; James D Bryers, I. B. Bioengineering report. Fouling biofilm development: A process analysis. Biotechnol. Bioeng., Vol. XXIII, Pp. 1923-60 (1981). Biotechnol. Bioeng. 2009, 102, (2), 309, 310-47.

(23) Mitchell, A. C.; Phillips, A. J.; Hamilton, M. A.; Gerlach, R.; Hollis, W. K.; Kaszuba, J. P.; Cunningham, A. B. Resilience of planktonic and biofilm cultures to supercritical $\mathrm{CO}_{2}$. J. Supercrit. Fluids 2008, 47 (2), 318-325.

(24) Lovley, D. R.; Phillips, E. J. P.; Gorby, Y. A.; Landa, E. R Microbial reduction of uranium. Nature 1991, 350 (6317), 413-416.

(25) Banat, I. M. Biosurfactants production and possible uses in microbial enhanced oil recovery and oil pollution remediation: A review. Bioresour. Technol. 1995, 51 (1), 1-12.

(26) Carr, H. Y.; Purcell, E. M. Effects of diffusion on free precession in nuclear magnetic resonance experiments. Phys. Rev. 1954, 94 (3), 630-638.

(27) Venkataramanan, L.; Song, Y. Q.; Hurlimann, M. D. Solving Fredholm integrals of the first kind with tensor product structure in 2 and 2.5 dimensions. IEEE Trans. Signal Process. 2002, 50 (5), 10171026.

(28) Hills, B. P.; Wright, K. M.; Belton, P. S. Proton NMR-studies of chemical and diffusive exchange in carbohydrate systems. Mol. Phys. 1989, 67 (6), 1309-1326.

(29) Walsh, D. O.; Grunewald, E.; Turner, P.; Frid, I., Javelin: A slimhole and microhole NMR logging tool. In FastTimes, 2010; Vol. 15, pp 67-72.

(30) Franklin, M. J.; Nivens, D. E.; Weadge, J. T.; Howell, P. L. Biosynthesis of the Pseudomonas aeruginosa extracellular polysaccarides, alginate, Pel, and Psl. Frontiers in Microbiology 2011, 2 (167), 167-1-16.

(31) Grant, G. T.; Morris, E. R.; Rees, D. A.; Smith, P. J. C.; Thom, D. Biological interactions between polysaccharides and divalent cations - egg-box model. FEBS Lett. 1973, 32 (1), 195-198.

(32) Borchard, W.; Kenning, A.; Kapp, A.; Mayer, C. Phase diagram of the system sodium alginate/water: A model for biofilms. Int. J. Biol. Macromol. 2005, 35 (5), 247-256.

(33) Hills, B. P.; Cano, C.; Belton, P. S. Proton NMR relaxation studies of aqueous polysaccharide systems. Macromolecules 1991, 24 (10), 2944-2950. 